\title{
Evaluation Research of Urban Distribution System's Resource Integration based on Generalized Regression Neural Network
}

\author{
Chen Liang ${ }^{1,2}$, Dong $\mathrm{Mu}^{1}$ and ZhengqiangJiang ${ }^{2}$ \\ ${ }^{1}$ Beijing Jiaotong University, Beijing 100044, China \\ ${ }^{2}$ Beijing Wuzi University, Beijing 101149, China \\ 1liangchen727@sina.com, ${ }^{2}$ mueast@163.com, ${ }^{3}$ injt19891011@126.com ’
}

The paper conducted an in-depth research of urban distribution system's resource integration process; It built the evaluation index sy stem of this issue at the beginning. Later, it studied the normalization methods to these corresponding indicators. Then, it constructed the evaluation model of urban distribution system's resource integration based on generalized regression neural network. At the end of this Paper, it conducted a numerical experiment example and made certain conctusions. The innovation of this paper can be described as that the evaluation modet constructed in this paper provides an effective and convenient way to evaluate the urhardistribution system's resource integration process.

Keywords: Generalized regression neurafnetwork, urban distribution system, resource integration, evaluation

\section{Introductions}

Urban distribution system's (UDS') resource integration is a kind of dynamic operation and management system, which aims at developing enterprises' core competitiveness with the help of scientific systems and modern technology, especially the modern computer network technology. With the integration of UDS' resource, UDS can avoid the vicious circle between its resource input and its efficiency [1]. However, in order to get an effective integration of UDS' resource, we should not only get a good understanding of the relationship between the associated elements, but also have an exact understanding of UDS' resource integration stage, thus, an accarate, comprehensive and fast system evaluation is indispensable [2].

There are many traditional evaluation methods, such as Delphi, AHP, fuzzy comprehensive evaluation method and some other evaluation methods [3], these methods are relatively mature and it can solve many different systems with properties evaluation indexes. However, there are still some deficiencies of these method, they are either have a certain subjective one-sidedness, or excessive reliance on its raw data [4]. Generalized regression neural network (GRNN) is a kind of neural network, they are not only have the general advantages of neural network algorithm, such as nonlinear, unreliable on subjective and objective environment, but also can avoid the shortcomings of general neural network effectively, such as slow convergence, easy to fall into local optimum, weak classification ability and low learning rate $[5,6]$. Therefore, this paper selected the GRNN method to study the evaluation of Urban distribution system's resource integration. 


\section{The Evaluation Index System of UDS' Resource Integration}

\subsection{The construction principle of its evaluation index system}

In order to get the best evaluation results of UDS' resource integration process, the evaluation index system we constructed should reflect an actual integration statue of UDS' resource truthfully, comprehensively and systematically. For this purpose, we should follow the following 6 basic principles which we can see below when constructing the evaluation index system $[7,8]$.

1) Systemic principle

Systematic principle requires that we should consider the UDS' resource integration issue as a whole system when selecting the evaluation indexes. We should consider the whole system, including all the subsystems and all the components, as the exaluation object, not only just part of them.

\section{2) Hierarchical principle}

Hierarchical principle requires that we should consider the UDS resource integration issue as a systematic and structured problem when/selecting the evaluation indexes; we should consider the hierarchical principle when selecting the evaluation indexes.

3) Comprehensiveness principle

Comprehensiveness principle requires that the evaluation index system we selected should cover all elements of this issue, and it also requires that we should turn the single evaluation to overall evaluation, turn from inward evaluation to internal and external combination evaluation, turn short-term e aluation tolong-term evaluation.

4) Scientific principle

Scientific Principles refers to that the evaluation index system should be designed around the evaluation objectrves and it can earnestly avoid the indexes unscientific, It is the basic principle in selecting evaluation indexes.

\section{5) Composite princip}

Composite princtiple requires that the evaluation index system we selected should be clear definite, intuitiye, clear, and include appropriate number of indexes when evaluating UDS' resource integration.

6) Operability principle

Operability principle requires that the evaluation index system we selected should be easy to understand, accuracy and operable when evaluation UDS' resource integration, the evaluation index system should be easy to operate.

\subsection{The design of its evaluation index system}

A scientific evaluation index system is the basis and premise of its system evaluation operation, this chapter makes an in-depth research on its index system by Brainstorming and Delphi methods, which was based on the wealthy knowledge of UDS and systematic, the indexes we selected can be seen in table 1 . 


\subsection{The normalization methods of these indexes}

The evaluation index can be divided into 2 categories; there are quantitative index and qualitative index. In the system evaluation process, the qualitative index can also be divided into 4 categories, which are efficiency index, cost index, interval index and fixed-type index. The heterogeneity between these indexes determined that we must normalize these indexes when evaluating the system. If we use the initial value as the input of one index, the systematic evaluation will be not objective. Thus, there must be normalization [9].

Suppose there are $\mathrm{n}$ evaluation target in the system, and each target have $\mathrm{m}$ indexes, the value of each index can be expressed as $x_{i j}(i=1,2, \ldots, n ; j=1,2, \ldots, m)$. So these values canbe composed to a matrix, which can be expressed $a x_{i j}$. Suppose the value of these indexes range from $x_{j}^{\min }$ to $x_{j}^{\max }$.

Table 1. The evaluation index system of UDS resource integration

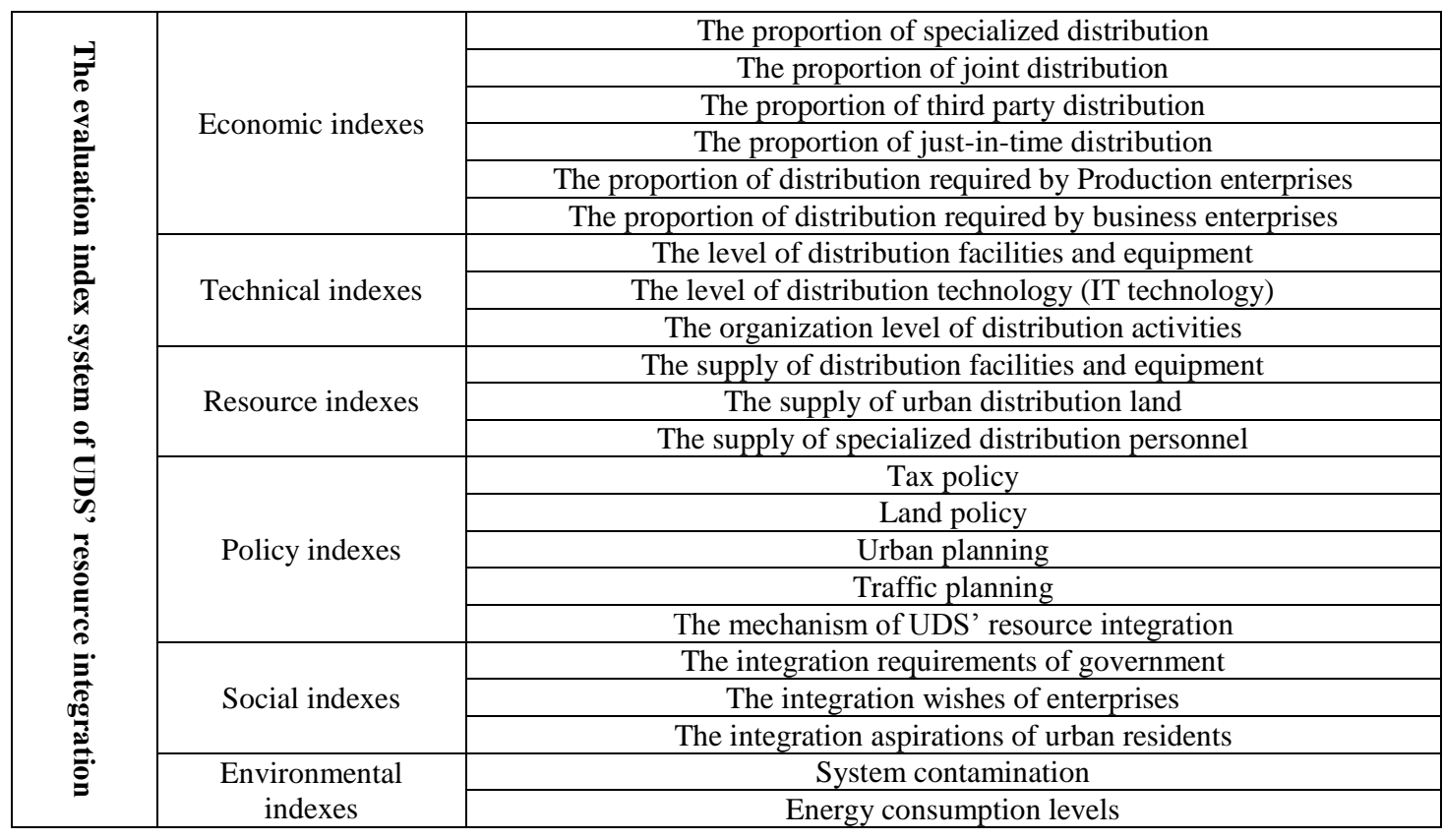

1) For the quantitative indexes

There are generally two kinds of indexes in system evaluation, which are efficiency index and cost index respectively, but also there are also another two kinds of indexes, which are intervalindex and fixed-type index.

(1)For the efficiency index, which means the bigger the value is, the better the index is. To these indexes, we can handle it as formula (1) below.

$$
Y_{i j}=\frac{x_{i j}-x_{j}^{\min }}{x_{j}^{\text {max }}-x_{j}^{\min }}
$$

(2)For the cost index, which means the bigger the value is, the worse the index is. To these indexes, we can handle it as formula (2) below.

$$
Y_{i j}=\frac{x_{j}^{\text {max }}-x_{i j}}{x_{j}^{\text {max }}-x_{j}^{\text {min }}}
$$


(3)For the interval index, which means the value of the index can neither too big nor to small, the value of the index in $\left[q_{\min }, q_{\max }\right]$ is the best.To these indexes, we can handle it as formula (3) below.

(4)For the fixed-type index, which means the value stable in certain value $x_{\text {best }}$, the index is the best, the greater the deviation from this fixed value is, the worse the index is. To these indexes, we can handle it as formula (4) below.

$$
\begin{gathered}
Y_{i j}=\left\{\begin{array}{l}
1-\frac{q_{\min }-x_{i j}}{\max \left(q_{\min }-x_{j}^{\text {min }}, x_{j}^{\text {max }}-q_{\max }\right)} x_{i j}<q_{\min } \\
1-\frac{x_{i j}-q_{\max }}{\max \left(q_{\min }-x_{j}^{\text {min }}, x_{j}^{\text {max }}-q_{\max }\right)} x_{i j}>q_{\max }
\end{array}\right. \\
Y_{i j}=\left\{\begin{array}{cc}
1 & x_{i j}=x_{\text {best }} \\
1-\frac{\left|x_{i j}-x_{\text {best }}\right|}{\max \left\{\left|x_{i j}-x_{\text {best }}\right|\right\}} x_{i j} \neq x_{b e s t}
\end{array}\right.
\end{gathered}
$$

2) For the qualitative index

For the qualitative indexes, it must be normalization only after being quantified first. For the quantitative, the most common used method is the expert scoring method. After calculating the scores, the indexes will be normalizedaccording to formula (1), (2), (3) and (4) after scoring by the relevant expefts and scholars. Then, we can get a matrix $Y_{i j}(i=$ $1,2, \ldots, \mathrm{n} ; \mathrm{j}=1,2, \ldots, \mathrm{m}$ which can be used to evaluate the system directly.

\section{The Evaluation Model Construction of UDS' Resource Integration}

For the basic theory of GRNN, there are a lot of discussions, and it can refer to references [10-12] specifically which 1 will not repeat here.

GRNN is a brand of neural network and it belongs to radial basis function (RBF) network. So the structure of GRNN is similar with RBF network, and they both have a linear input layer, a number of RBF hidden layers and a linear output layer [13]. The specific structure can be seen in Figure 1

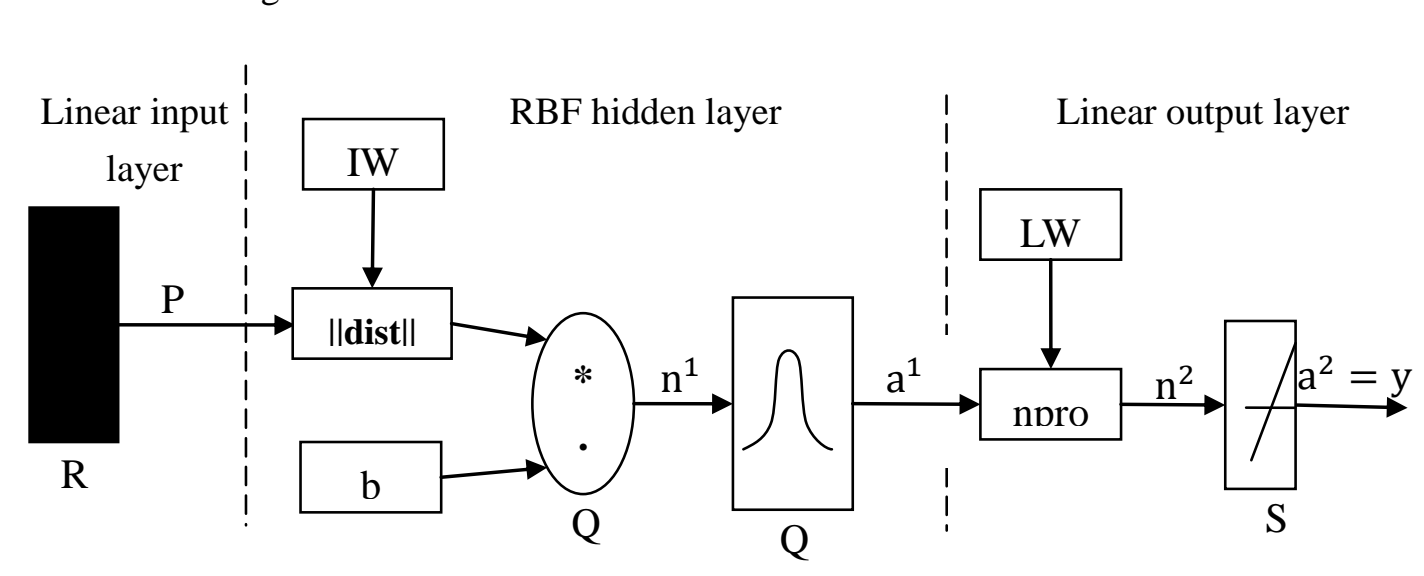

Figure 1. The structure graph of GRNN 
The first layer of this network is linear input layer of the variables, it is only responsible for processing the normalized values to the hidden layer, and it doesn't participate in any calculations.

The second layer of this network is RBF hidden layers, there can be one hidden layer, and also there can be several hidden layers, which is responsible for the calculation of these variables. The number of neurons in this layer must be equal to the number of indexes, and the weight function in this layer is the Euclidean distance function (shown in formula (5) by\|dist $\|$ ), which aims at calculating the distance between the input layer and hidden layerIW. The symbolb represents the threshold of hidden layer, which shows in figure 1 indicate by the symbol "•". We generally use Gaussian function (see equation (6)) as the transfer function, as the Gaussian function's axisymmetic and central projection characteristic, which makes the hidden layer produces a larger output when the input value is close to the middle of Gaussian function, this feature makes Gaussian function a good local approximation capability, which is essential important to improve the learning efficiency of GRNN.

$$
\begin{gathered}
\| \text { dist }\|=\| \mathrm{IW}-\mathrm{P} \|=\sqrt{\sum_{\mathrm{i}=1}^{\mathrm{R}}\left(\mathrm{I} W_{\mathrm{t}}-\mathrm{P}\right)^{2}} \\
\mathrm{R}_{\mathrm{i}}(\mathrm{x})=\exp \left(-\frac{\left\|\mathrm{x}-\mathrm{x}_{\mathrm{i}}\right\|^{2}}{2 \delta_{\mathrm{i}}^{2}}\right)^{\circ}
\end{gathered}
$$

The third layer of this network is linear output Payer, and the weight function is the normalized dot product function (shøen in figure, $\mathbf{N}$ by nprod), this formula is to achieve the normalization of LW and $\mathrm{a}^{1}$ (refers lo equatation (7)), equation (8) is used to output the evaluation result.

$$
\begin{gathered}
n^{i}=\operatorname{nomprod}\left(L W_{2,1} a^{i}\right)=\frac{L W_{2,1} * a^{i}}{\sum_{j=1}^{Q} a_{j}^{i}} ; i=1,2, \ldots, Q \\
a^{i}=y-\operatorname{purelin}\left(n^{i}\right)=n^{i} ; i=1,2, \ldots, Q
\end{gathered}
$$

The learning algorithm of GRNN is similar with RBF network, the only difference in the linear output layer. The learning algorithm of its linear output layer shows in equations (7) and equation (8).

There are four advantages of GRNN compared with general neural network algorithm which can be seen as follows, and these four advantages lead to select GRNN as the evaluation method of UDS' resource integration.

1) The raming category of this network is one-way training, which means there is no iteration. It can not only reduce the difficulty of the algorithm, but also improve the efficiency.

2) The number of neurons is determined by the training samples, and this value has already been proven to be the most effective value, which eliminate the tedious neurons testing.

3) The weights between each layer are uniquely determined by the training samples, and they avoid modifying the weights, which can improve the algorithm's learning efficiency.

4) The activation function used in the RBF hidden layers is Gaussian function, which has the local activation characteristics of the input values, and it has a strong attraction of the input values which can help improving the learning efficiency. 
In the evaluation process of GRNN, it can be divided into the following six steps according to its evaluation purpose and requirement, and the specific steps can be seen in Figure 2 listed as below.

the training set and test set

$\longrightarrow$ Create GRNN $\longrightarrow$ Model Simulation

Final model determination Smooth-factor selection Performance Evaluation

Figure 2. The evaluation process of GRNN

1) Generate the training set and test set

In the process of training set and test set generating, we should not)onty consider the scale of training set sample and test set sample, but also analyze the fidelity and reliability of these samples. The specific procedares to generate the training set and test set are as follows:

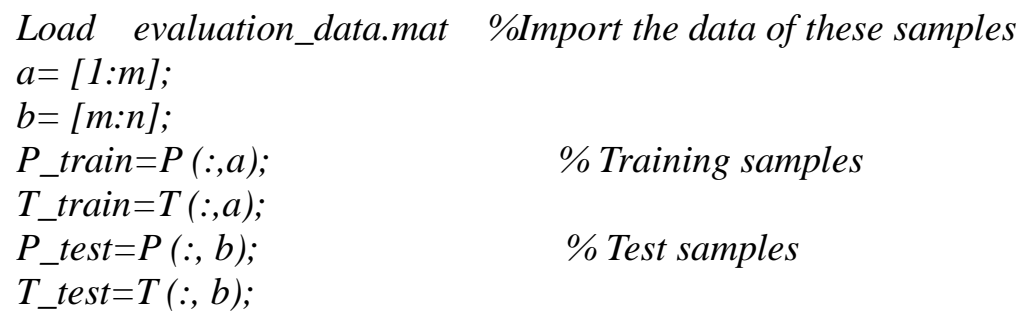

2) Creat the GRNN

We create the GRNN by MATAAB R2010a software, and the specific procedures can be seen as follow:

Net $\operatorname{grnn}=n e t g r n n\left(P \_t r a i n, T \_t r a i n, s p r e a d\right)$;

$\%$ spread represent the smooth coefficient indicates

3) Model simulation

After creating the GRNN, we can import the normalized data into the model, and the model can be self-output, the output value is the corresponding test results. The specific procedures are as follows:

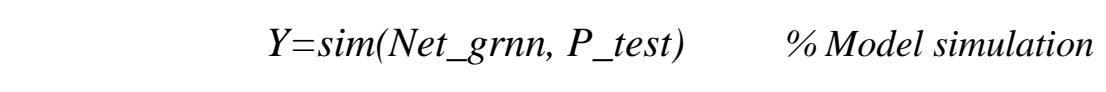

\section{4) Performance Evaluation}

Evaluate the effect of this model by calculating the output data and its actual date. If the effect performs poor, test the smooth-factors.

\section{5) Smooth-factor selection}

The smooth-factor values $(0.1,0.1,0.9)$, we carried out 9 experiments and calculate the errors between the predicting data and its actual data (shown in formula (9)). So we can select the least error data which also means the best-performed data as the model's smooth-factor value. The error of each data can be calculated as formula (9). 


$$
\text { \%error }=\frac{Y_{i j}-y_{i j}}{y_{i j}} \%
$$

6) Final model determination

After determining the smooth-factor, the final evaluation model has been established, and we can use this model to evaluate the UDS' resource integration process.

\section{Numerical experiments}

\subsection{Data initialization}

The numerical experiment has six samples, and it carries out the evaluation research of UDS' resource integration based on GRNN, expect to provide a scientificlevaluation model for UDS' resource integration. Table 2 shows the yalue of standard normalized dimensionless index $\left(\mathrm{Y}_{\mathrm{k}}\right.$ corresponding $\mathrm{X}_{\mathrm{k}}$ respectively) and table 3 shows the output sample data.

\subsection{Network parameters selection}

During the network parameters selection process, it mainly related to the nodes number of input leaner layer and the walue of smooth factor. The evaluation index system includes 22 indexes, so the number of nodes is 22. While, to the smooth factor, it values as $(0.1: 0.1: 1)$ respectively. 2 fter all these parameters are determined, we carry out a comparative analysis of the output results.

Table 2. Normalized index characteristic values with the non-dimensional

\begin{tabular}{|c|c|c|c|c|c|c|c|c|c|c|c|}
\hline & $\mathbf{Y}_{\mathbf{1}}$ & $\mathbb{Y}_{\mathbf{2}}$ & $\mathbf{Y}_{\mathbf{3}}$ & $\mathbf{Y}_{\mathbf{4}}$ & $\mathbf{Y}_{\mathbf{5}}$ & $\mathbf{Y}_{\mathbf{6}}$ & $\mathbf{Y}_{\mathbf{7}}$ & $\mathbf{Y}_{\mathbf{8}}$ & $\mathbf{Y}_{\mathbf{9}}$ & $\mathbf{Y}_{\mathbf{1 0}}$ & $\mathbf{Y}_{\mathbf{1 1}}$ \\
\hline Sample 1 & 0.125 & 0.667 & 1 & 0 & 0.25 & 0 & 0.667 & 0.667 & 0.333 & 1 & 1 \\
\hline Sample 2 & 0 & 0.11 & 0 & 0.3 & 1 & 0.556 & 1 & 1 & 0.667 & 0 & 0 \\
\hline Sample 3 & 0.5 & $\mathbf{Y}$ & 0.308 & 0.5 & 0.625 & 0 & 0 & 0 & 0.333 & 0.5 & 0 \\
\hline Sample 4 & & 0.556 & 0.385 & 0.8 & 0.25 & 0.889 & 0 & 0.333 & 0.333 & 0.25 & 0.333 \\
\hline Sample 5 & 0.25 & 0 & 0.615 & 1 & 0 & 1 & 0.333 & 0.333 & 1 & 0.75 & 0.667 \\
\hline Sample 6 & 0.375 & 0.444 & 0.462 & 0.4 & 0.375 & 0.667 & 0.667 & 1 & 0 & 0.5 & 0 \\
\hline & $\mathbf{Y}_{\mathbf{1 2}}$ & $\mathbf{Y}_{\mathbf{1 3}}$ & $\mathbf{Y}_{\mathbf{1 4}}$ & $\mathbf{Y}_{\mathbf{1 5}}$ & $\mathbf{Y}_{\mathbf{1 6}}$ & $\mathbf{Y}_{\mathbf{1 7}}$ & $\mathbf{Y}_{\mathbf{1 8}}$ & $\mathbf{Y}_{\mathbf{1 9}}$ & $\mathbf{Y}_{\mathbf{2 0}}$ & $\mathbf{Y}_{\mathbf{2 1}}$ & $\mathbf{Y}_{\mathbf{2 2}}$ \\
\hline Sample 1 & 0.75 & 0.667 & 1 & 1 & 1 & 0 & 0.4 & 0 & 1 & 0 & 0 \\
\hline Sample 2 & 05 & 0 & 0.5 & 0.75 & 0.25 & 0.5 & 0.8 & 0.333 & 0.4 & 0.667 & 0 \\
\hline Sample 3 & 05 & 1 & 1 & 0 & 0.25 & 1 & 1 & 1 & 0.4 & 0.333 & 0.5 \\
\hline Sample 4 & 025 & 0.333 & 0 & 0.25 & 0.5 & 0.5 & 0.6 & 0.333 & 0.6 & 0.667 & 1 \\
\hline Sample 5 & 0 & 0 & 0.5 & 0.75 & 0.75 & 0 & 0 & 0 & 0.8 & 1 & 1 \\
\hline Sanple6 & 1 & 0.667 & 1 & 0.5 & 0.25 & 0 & 0.2 & 0.333 & 0.4 & 1 & 0.5 \\
\hline
\end{tabular}

Table 3. The actual data of the samples

\begin{tabular}{cccccc}
\hline \multicolumn{6}{c}{ Samples } \\
\hline 1 & 2 & 3 & 4 & 5 & 6 \\
\hline 9.12 & 7.25 & 8.64 & 8.38 & 7.51 & 8.37 \\
\hline Note: & These actual data is obtained by Delphi
\end{tabular}

\subsection{Sample testing and experimental results analyzing}

We take the samples No. 1-4 as training samples, and No. 5-6 as the testing sample. This paper select the GRNN toolbox of MATLAB R2010a to evaluate the UDS' 
resource integration, and the errors of training samples and test samples varies with different smooth factor $\alpha$. The errors of training samples and test samples calculated by GRNN can be seen in Figure 3.

As we can see from Figure 3, the smaller the smooth-factor is the smaller error of training samples and test samples. When the smooth-factor is between 0.1 and 0.2 , the evaluating results are able to meet the evaluating requirement, not only to the approximation performance, but also to test performance. Therefore, the smooth-factor can be valued $(0,0.2]$ when determining the final evaluation model.

As we can see from the previous numerical experiment, this evaluation model is feasibility in evaluating the UDS' resource integration, and it provides an effective evaluation tool for UDS' resource integration. And as the programming of GRXN is simple, and there are little parameters need to adjust; it has a strong Competitive advantage in evaluating the UDS' resource integration.

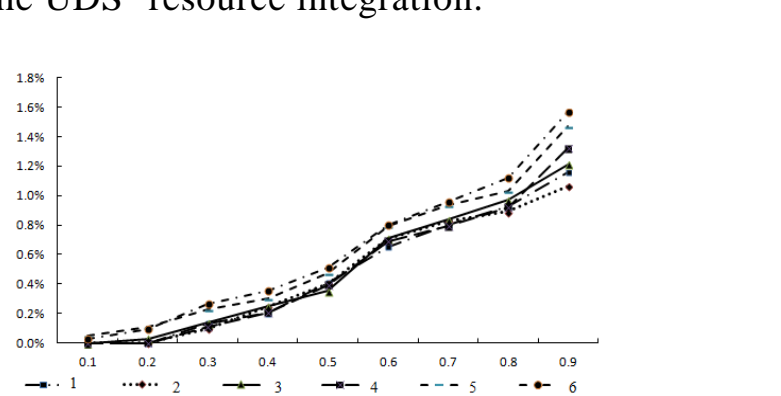

Note: Line 1, 2, 3 and 4 represent the er@or of training samples and line 5 and 6 represents the error of testing samples

Figure 3. The errors of training samples and test samples calculated by GRNN

\section{Conclusions}

This paper consfructed the evaluation index system of UDS' resource integration at first, after that, it designed the evaluation model by GRNN. The Numerical experiment proved the validity of thi model at the end of this paper. In conclusion, the main findings of this paper can be summarized as the following 2 points: 1) It constructs the evaluation model of UDS' resource integration; 2) It established the evaluation model of UDS' resource integration based on GRNN.

\section{Acknowledgements}

The research work was supported by National Natural Science Foundation of China under Grant No. 71132008, Funding Project for Academic Human Resources Development in Institutions of Higher Learning under the Jurisdiction of Beijing Municipality and the Scientific Research Bases-Science and technology innovation platform of Beijing Wuzi University.

\section{References}

[1] C. Liang, "The formation mechanism and complexity research of central urban distribution system", China Logistics and Purchasing, no. 24, (2011), pp. 70-71.

[2] A. Zheng, "City distribution system optimization research", Wuhan University of Technology, (2005).

[3] Y. Zhang and D. Liu, "Summary of studies on performance evaluation of logistics enterprises", Logistics Technology, vol. 29, no. 008, (2010), pp. 14-16.

[4] J. Liu, T. Zhou, Q. Guo, et al., "Overview of the evaluated algorithms for the personal recommendation systems”, Complex System and Complexity Science, vol. 6, no. 3, (2009), pp. 1-10. 
[5] Z. Leng, J. Gao, Y. Qin, et al., "Short-term forecasting model of traffic flow based on GRNN", Control and Decision Conference (CCDC), (2013) 25th Chinese, IEEE 2013, pp. 3816-3820.

[6] C. Lopez-Martin, C. Isaza and A. Chavoya, "Software development effort prediction of industrial projects applying a general regression neural network", Empirical Software Engineering, vol. 17, no. 6, (2012), pp. 738-756.

[7] Y. Sun, K. Xu and W. Qin, "Risk assessment of project contractors based on generalized regression neural network", Industrial Engineering Journal, vol. 14, no. 3, (2011), pp. 119-123.

[8] J. Liu, "On the Firms' Evolutionary Mechanisms Based on the Theory of Self-organization", Harbin Engineering University, (2005).

[9] N. E. Gronlumd and R. L. Linn, "Measurement and evaluation in teaching", (2012).

[10] K. Zhu and Z. Wang, "Proficient in MATLAB neural network", Electronic Industry Press, (2010).

[11] P. V. B. Reddy, C. Kumar and K. H. Reddy, "Modeling of wire EDM process using back propagation (BPN) and General Regression Neural Networks (GRNN)", Frontiers in Automobile and Mechanical Engineering (FAME), (2010), IEEE 2010, pp. 317-321.

[12] Y. Jia, Q. Lv and Y. Shang, "Rock Burst Prediction Using Particle Swarm Optimization algorithm and General Regression Neural Network", Chinese Journal of Rock Mechanics and Engineering, vol. 32, no. 2, (2013), pp. 343-348.

[13] S. C. Yang, C. S. Lee and H. S. Lee, "Evaluation of Logistic Flow Service Sat sfaction Using The Evolutionary Computation Technique And General Regression Neural Network Technique", International Journal of Advancements in Computing Technology, vol. 4, no 1), (2013)

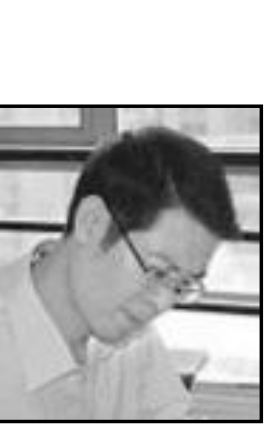

\section{Chen Liang}

\section{Authors}

He is a full instructor of Beijing Wuzi University (2005). He received his master degree ih Management science and engineering from Beijing Wuzi University, and now he is working on his $\mathrm{PhD}$ degree in Beijing Jiaotong University science 2009. His current research interests include different aspects of logrstics engineering and Artificial Intelligence and Systems dynamigs.

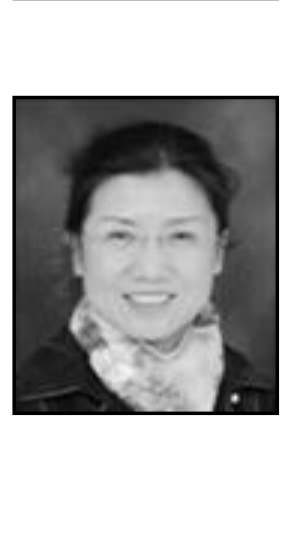

\section{Dong Mu}

She received her Ph.D degree in Business and Management School of Tong J University (1999). Now she is a full time professor at Management School of Beijing Jiaotong University since 2005. Her current research interests include different aspects of logistics and supply system engineering, regional logistics and environmental evaluation and system dynamics.

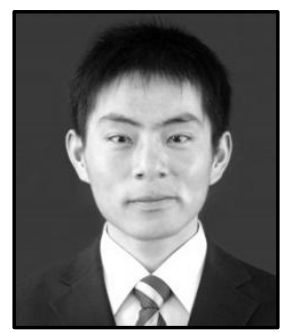

\section{Zhengqiang Jiang}

$\mathrm{He}$ is a full master student of Beijing Wuzi University (2011). He received his undergraduate diploma in Huazhong University of Science and Technology, and now he is work on his master. His current research interests include different aspects of logistics engineering and Artificial Intelligence and Systems dynamics. 
International Journal of Multimedia and Ubiquitous Engineering

Vol.9, No.4 (2014)

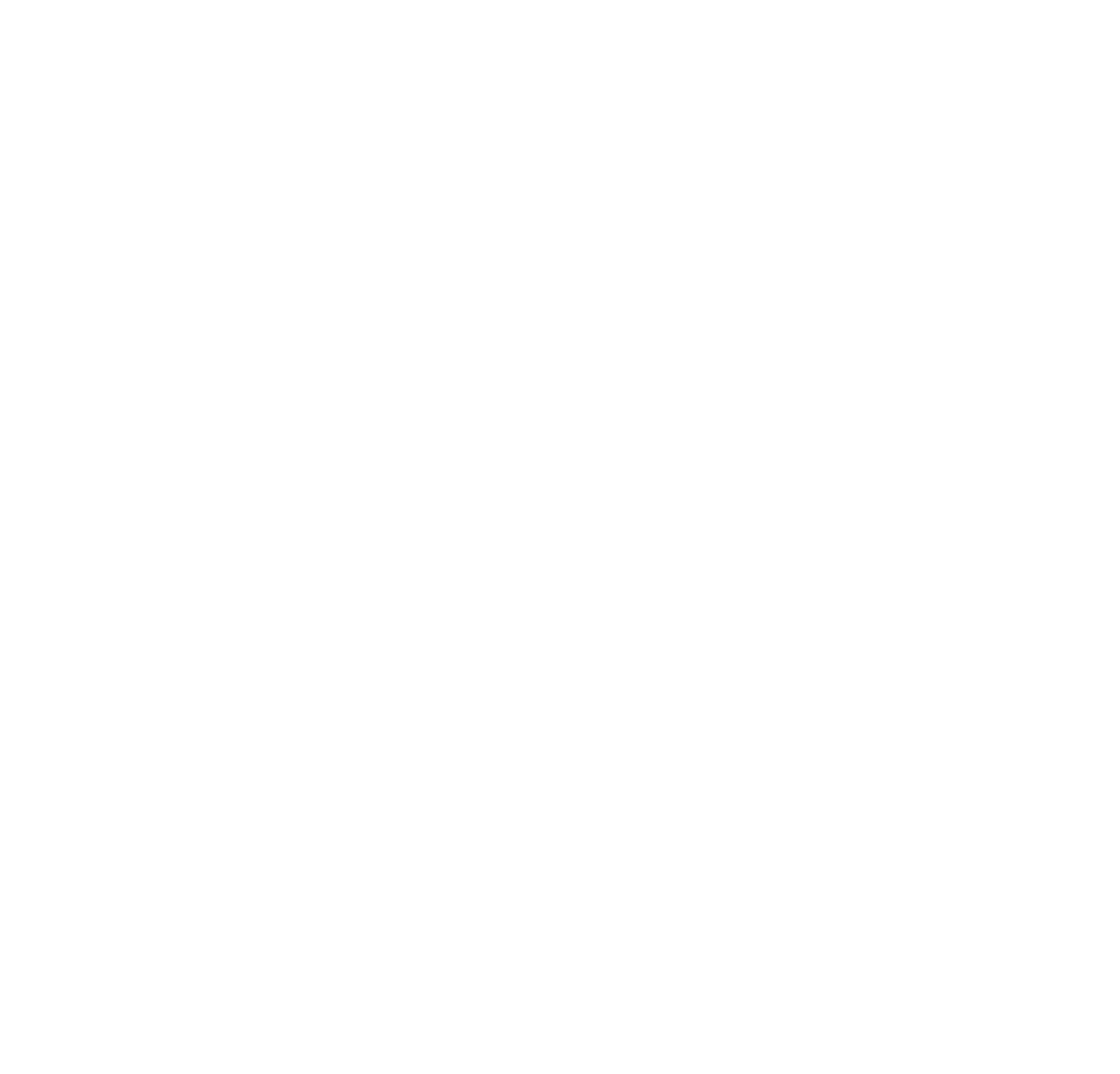

